\title{
Grassmann analysis: basics
}

\subsection{Introduction}

Parity is ubiquitous, and Grassmann analysis is a tool well adapted for handling systematically parity and its implications in all branches of algebra, analysis, geometry and topology. Parity describes the behavior of a product under exchange of its two factors. The so-called Koszul's parity rule states: "Whenever you interchange two factors of parity 1 ,

you get a minus sign". Formally the rule defines graded commutative products.

$$
A B=(-1)^{\tilde{A} \tilde{B}} B A
$$

where $\tilde{A} \in\{0,1\}$ denotes the parity of $A$. Objects with parity zero are called even, and objects with parity one odd. The rule also defines graded anticommutative products. For instance,

$$
A \wedge B=-(-1)^{\tilde{A} \tilde{B}} B \wedge A .
$$

A graded commutative product $[A, B]$ can be either a commutator $[A, B]_{-}$, or an anticommutator $[A, B]_{+}$.

A graded anticommutative product $\{A, B\}$ can be either an anticommutator $\{A, B\}_{+}$, or a commutator $\{A, B\}_{-}$. Most often, the context makes it unnecessary to use the + and - signs.

There are no (anti)commutative rules for vectors and matrices. Parity is assigned to such objects in the following way.

- The parity of a vector is determined by its behavior under multiplication with a scalar $z$ :

$$
z X=(-1)^{\tilde{z} \tilde{X}} X z
$$


- A matrix is even if it preserves the parity of graded vectors and odd if it inverts the parity.

Vectors and matrices do not necessarily have well-defined parity, but they can always be decomposed into a sum of even and odd parts.

The usefulness of Grassmann analysis in physics became apparent in the works of F.A. Berezin [1], and M.S. Marinov [2]. We refer the reader to $[3],[4],[5],[6]$, and [7] for references and recent developments. The next section summarizes the main formulae of Grassmann analysis.

As a rule of thumb, it is most ofen sufficient to insert the word "graded" in the corresponding ordinary situation. For example an ordinary form is an antisymmetric covariant tensor, a Grassmann form is a graded antisymmetric covariant tensor: $\omega_{\ldots \alpha \beta \ldots}=-(-1)^{\tilde{\alpha} \tilde{\beta}} \omega_{\ldots \beta \alpha \ldots}$ where $\tilde{\alpha} \in\{0,1\}$ is the grading of $\alpha$. Therefore a Grassmann form is symmetric in the interchange of two Grassmann indices.

\subsection{A compendium of Grassmann analysis}

This section is extracted from the Master Thesis [8] of Maria E. Bell "Introduction to Supersymmetry". We collect here formulae which are selfexplanatory as well as formulae whose meaning is given in the following sections, in order that all symbols be located in the same section.

Basic graded algebra, a dictionary

- $\tilde{A}:=$ parity of $A \in\{0,1\}$

- Parity of a product:

$$
\widetilde{A B}=\tilde{A}+\tilde{B} \bmod 2
$$

- Graded commutator:

$$
[A, B]:=A B-(-1)^{\tilde{A} \tilde{B}} B A \quad \text { or } \quad[A, B]_{\mp}=A B \mp B A
$$

- Graded anticommutator:

$$
\{A, B\}:=A B+(-1)^{\tilde{A} \tilde{B}} B A \quad \text { or } \quad\{A, B\}_{ \pm}=A B \pm B A
$$

- Graded Leibnitz rule for a differential operator:

$$
D(A \cdot B)=D A \cdot B+(-1)^{\tilde{A} \tilde{D}}(A \cdot D B)
$$

(previously called "antileibnitz" when $\tilde{D}=1$ ). 
- Graded symmetry:

$$
A^{\cdots \alpha \beta \ldots}=(-1)^{\tilde{\alpha} \tilde{\beta}} A^{\cdots \beta \alpha \cdots}
$$

- Graded antisymmetry:

$$
A^{\cdots \alpha \beta \ldots}=-(-1)^{\tilde{\alpha} \tilde{\beta}} A^{\cdots \beta \alpha \ldots}
$$

- Graded Lie derivative:

$$
\mathcal{L}_{X}=\left[i_{X}, d\right]_{+} \text {for } \tilde{X}=0, \quad \text { and } \quad \mathcal{L}_{\Xi}=\left[i_{\Xi}, d\right]_{-} \text {for } \tilde{\Xi}=1 .
$$

- Grassmann generators $\left\{\xi^{\mu}\right\} \in \Lambda_{\nu}, \Lambda_{\infty}, \Lambda$

$$
\xi^{\mu} \xi^{\sigma}=-\xi^{\sigma} \xi^{\mu} ; \Lambda=\Lambda^{\text {even }} \oplus \Lambda^{\text {odd }}
$$

- Supernumber $z=u+v, u$ is even, $v$ is odd

$$
z=z_{B}+z_{S}, z_{B} \in \mathbb{R} \text { is the body, } z_{S} \text { is the soul }
$$

- Complex conjugation of supernumber:

$$
\left(z z^{\prime}\right)^{*}=z^{*} z^{*} .
$$

Complex conjugation is also defined $\left(z z^{\prime}\right)^{*}=z^{*} z^{*}$. We prefer the definition (9.13) for the following reason [5]: Let a supernumber

$$
\psi=c_{0}+c_{i} \xi^{i}+\frac{1}{2 !} c_{i j} \xi^{i} \xi^{j}+\ldots
$$

be called real if all its coefficients $c_{i_{1} \ldots i_{p}}$ are real numbers, and let

$$
\psi=\rho+i \sigma
$$

where both $\rho$ and $\sigma$ have real coefficients. Define complex conjugation by

$$
(\rho+i \sigma)^{*}=\rho-i \sigma .
$$

Then the generators $\left\{\xi^{i}\right\}$ are real, and the sum and product of 2 real supernumbers are real. Furthermore

$$
\psi \text { is real } \Leftrightarrow \psi^{*}=\psi .
$$

With the other definition of complex conjugation, the product of two real supernumbers is purely imaginary. 
- Superpoints. Real coordinates $x, y \in \mathbb{R}^{n}, x=\left(x^{1}, \ldots, x^{n}\right)$

$\left(x^{1}, \ldots, x^{n}, \xi^{1}, \ldots, \xi^{\nu}\right) \in \mathbb{R}^{n \mid \nu}$, condensed notation $x^{A}=\left(x^{a}, \xi^{\alpha}\right)$

$$
\left(u^{1}, \ldots, u^{n}, v^{1}, \ldots, v^{\nu}\right) \in \mathbb{R}_{c}^{n} \times \mathbb{R}_{a}^{\nu} .
$$

- Supervectorspace, i.e. a graded module over the ring of supernumbers

$$
\begin{aligned}
X & =U+V, U \text { even, } V \text { odd } \\
X & =e_{(A)}{ }^{A} X \\
X^{A} & =(-1)^{\tilde{X} \tilde{A}}{ }^{A} X .
\end{aligned}
$$

The even elements of the basis $\left(e_{(A)}\right)_{A}$ are listed first. A supervector is even if each of its coordinates ${ }^{A} X$ has the same parity as the corresponding basis element $e_{(A)}$. It is odd if the parity of ${ }^{A} X$ is opposite to the parity of $e_{(A)}$. Parity cannot be assigned in other cases.

- Graded Matrices. Four different uses of graded matrices:

given $V=e_{(A)}{ }^{A} V=\bar{e}_{(B)}{ }^{B} \bar{V}$ with $A=(a, \alpha)$ and $e_{(A)}=\bar{e}_{(B)}{ }^{B} M_{A}$ then ${ }^{B} \bar{V}={ }^{B} M_{A}{ }^{A} V$

given $\langle\omega, V\rangle=\omega_{A}{ }^{A} V=\bar{\omega}_{B}{ }^{B} \bar{V}$ where $\omega=\omega_{A}{ }^{(A)} \theta=\bar{\omega}_{B}{ }^{(B)} \bar{\theta}$ then $\langle\omega, V\rangle=\omega_{A}\left\langle{ }^{(A)} \theta, e_{(B)}\right\rangle{ }^{B} V$ implies $\left\langle{ }^{(A)} \theta, e_{(B)}\right\rangle={ }^{A} \delta_{B}$, $\omega_{A}=\bar{\omega}_{B}{ }^{B} M_{A}$, and ${ }^{(B)} \bar{\theta}={ }^{B} M_{A}{ }^{(A)} \theta$.

- Matrix parity:

$$
\begin{aligned}
& \tilde{M}=0 \text {, if } \forall A \text { and } B, \widetilde{B_{A}}+\widetilde{\operatorname{column}} B+\widetilde{\operatorname{row} A}=0 \bmod 2 . \\
& \tilde{M}=1 \text {, if } \forall A \text { and } B, \widetilde{B_{M_{A}}}+\widetilde{\operatorname{column}} B+\widetilde{\operatorname{row} A}=1 \bmod 2 .
\end{aligned}
$$

By multiplication, an even matrix preserves the parity of the vector components, an odd matrix inverts the parity of the vector components.

- Supertranspose:

$$
(M N)^{\mathrm{ST}}=(-1)^{\tilde{M} \tilde{N}} N^{\mathrm{ST}} M^{\mathrm{ST}} .
$$

- Superhermitian conjugate:

$$
\begin{gathered}
M^{\mathrm{SH}}:=\left(M^{\mathrm{ST}}\right)^{*}=\left(M^{*}\right)^{\mathrm{ST}} \\
(M N)^{\mathrm{SH}}=(-1)^{\tilde{M} \tilde{N}} N^{\mathrm{SH}} M^{\mathrm{SH}} .
\end{gathered}
$$

- Graded operators on Hilbert spaces. An operator and its eigenvalues 
have the same parity. Let $|\Omega\rangle$ be a simultaneous eigenstate of $Z$ and $Z^{\prime}$ with eigenvalues $z$ and $z^{\prime}$ :

$$
\begin{gathered}
Z Z^{\prime}|\Omega\rangle=z z^{\prime}|\Omega\rangle \\
\langle\Omega| Z^{\prime \mathrm{SH}} Z^{\mathrm{SH}}=\langle\Omega| z^{\prime *} z^{*} .
\end{gathered}
$$

- Supertrace:

$$
\operatorname{Str} M=(-1)^{\tilde{A} A} M_{A} .
$$

Example. A matrix of order $(p, q)$. Assume the $p$ even rows and columns written first
$M_{0}=($
)$=\left(\begin{array}{cc}A_{0} & C_{1} \\ D_{1} & B_{0}\end{array}\right) \quad M_{1}=($
)$=\left(\begin{array}{ll}A_{1} & C_{0} \\ D_{0} & B_{1}\end{array}\right)$

These are two matrices of order $(1,2)$. The shaded areas cover even elements. The matrix on the left is even; the one on the right is odd.

$$
\operatorname{Str} M_{0}=\operatorname{tr} A_{0}-\operatorname{tr} B_{0} ; \quad \operatorname{Str} M_{1}=-\operatorname{tr} A_{1}+\operatorname{tr} B_{1} .
$$

- Super determinant (a.k.a. Berezinian). It is defined so that it satisfies the basic properties

$$
\begin{gathered}
\operatorname{Ber} M N=\operatorname{Ber} M \operatorname{Ber} N \\
\delta \ln \operatorname{Ber} M=\operatorname{Str}\left(M^{-1} \delta M\right) \\
\operatorname{Ber} \exp M=\exp \operatorname{Str} M \\
\operatorname{Ber}\left(\begin{array}{cc}
A & C \\
D & B
\end{array}\right):=\operatorname{det}\left(A-C B^{-1} D\right)(\operatorname{det} B)^{-1} .
\end{gathered}
$$

- Parity assignments:

$$
\tilde{d}=1 \quad(\widetilde{d x})=\tilde{d}+\tilde{x}=1 \quad(\tilde{d} \xi)=\tilde{d}+\tilde{\xi}=0,
$$

$x$ ordinary variable, $\xi$ Grassmann variable,

$$
(\widetilde{\partial / \partial x})=\tilde{x}=0 \quad(\widetilde{\partial / \partial \xi})=\tilde{\xi}=1
$$




$$
\tilde{i}=1 \quad \widetilde{i_{X}}=\tilde{i}+\tilde{X}=1 \quad \tilde{i_{\Xi}}=\tilde{i}+\tilde{\Xi}=0 \text {. }
$$

Parity of real $p$-forms: even for $p=0 \bmod 2$, odd for $p=1 \bmod 2$ Parity of Grassmann $p$-forms: always even.

Graded exterior product $\omega \wedge \eta=(-1)^{\tilde{\omega} \tilde{\eta}} \eta \wedge \omega$.

Forms and densities will be introduced in Section 9.4. In the following four entries, we list definitions and properties of objects defined on ordinary manifolds $\mathbb{M}^{D}$, first without metric tensor, then on riemannian manifolds $\left(\mathbb{M}^{D}, g\right)$.

Forms and densities of weight one on an ordinary manifolds $\mathbb{M}^{D}$

(without metric tensor)

$\left(\mathcal{A}^{\bullet}, d\right)$ Ascending complex of forms $d: \mathcal{A}^{p} \rightarrow \mathcal{A}^{p+1}$

$\left(\mathcal{D}_{\bullet}, \nabla \cdot\right.$ or $\left.b\right)$ Descending complex of densities $\nabla \cdot: \mathcal{D}_{p} \rightarrow \mathcal{D}_{p-1}$

$\mathcal{D}_{p} \equiv \mathcal{D}^{-p}$ used for ascending complex in negative degrees.

- Operators on $\mathcal{A}^{\bullet}\left(\mathbb{M}^{D}\right)$ :

$M(f): \mathcal{A}^{p} \rightarrow \mathcal{A}^{p}$, multiplication by a scalar function $f: \mathbb{M}^{D} \rightarrow \mathbb{R}$

$e(f): \mathcal{A}^{p} \rightarrow \mathcal{A}^{p+1}$ by $\omega \mapsto d f \wedge \omega$

$i(X): \mathcal{A}^{p} \rightarrow \mathcal{A}^{p-1}$ by contraction with the vectorfield $X$

$\mathcal{L}_{X} \equiv \mathcal{L}(X)=i(X) d+d i(X): \mathcal{A}^{p} \rightarrow \mathcal{A}^{p}$ by the Lie derivative w.r.t. $X$.

- Operators on $\mathcal{D}_{\bullet}\left(\mathbb{M}^{D}\right)$ :

$M(f): \mathcal{D}_{p} \rightarrow \mathcal{D}_{p}$, multiplication by scalar function $f: \mathbb{M}^{D} \rightarrow \mathbb{R}$

$e(f): \mathcal{D}_{p} \rightarrow \mathcal{D}_{p-1}$ by $\mathcal{F} \rightarrow d f \cdot \mathcal{F}$ (contraction with the form $d f$ )

$i(X): \mathcal{D}_{p} \rightarrow \mathcal{D}_{p+1}$ by multiplication and partial antisymmetrization

$\mathcal{L}_{X} \equiv \mathcal{L}(X)=i(X) \nabla+\nabla i(X): \mathcal{D}_{p} \rightarrow \mathcal{D}_{p}$ by Lie derivative w.r.t. $X$.

- Forms and densities of weight one on $\left(\mathbb{M}^{D}, g\right)$ :

$C_{g}: \mathcal{A}^{p} \rightarrow \mathcal{D}_{p}$ (see eq. (9.59))

$*: \mathcal{A}^{p} \rightarrow \mathcal{A}^{D-p}$ s.t. $\mathcal{T}(\omega \mid \eta)=\omega \wedge * \eta$

$\delta: \mathcal{A}^{p+1} \rightarrow \mathcal{A}^{p}$ is the metric transpose defined by

$$
[d \omega \mid \eta]=:[\omega \mid \delta \eta] \text { s.t. }[\omega \mid \eta]:=\int \mathcal{T}(\omega \mid \eta)
$$

$\delta=C_{g}^{-1} b C_{g}$ (see eq. (9.66))

$\beta: \mathcal{D}_{p+1} \rightarrow \mathcal{D}_{p}$ is defined by $C_{g} d C_{g}^{-1}$.

In the following four entries we list definitions and properties of objects defined on Grassmann variables. 
Grassmann calculus on $\xi^{\lambda} \in \Lambda_{\nu}$ or $\Lambda_{\infty}$ or unspecified $\Lambda$

$d d=0$ remains true, therefore

$$
\begin{aligned}
\frac{\partial}{\partial \xi^{\lambda}} \frac{\partial}{\partial \xi^{\mu}} & =-\frac{\partial}{\partial \xi^{\mu}} \frac{\partial}{\partial \xi^{\lambda}} \\
d \xi^{\lambda} \wedge d \xi^{\mu} & =d \xi^{\mu} \wedge d \xi^{\lambda} .
\end{aligned}
$$

- Forms and densities of weight -1 on $\mathbb{R}^{0 \mid \nu}$ :

Forms are graded totally symmetric covariant tensors. Densities are graded totally symmetric contravariant tensors of weight -1 . $\left(\mathcal{A}^{\bullet}\left(\mathbb{R}^{0 \mid \nu}\right), d\right)$ Ascending complex of forms not limited above $\left(\mathcal{D} \cdot\left(\mathbb{R}^{0 \mid \nu}\right), \nabla \cdot\right.$ or $\left.b\right)$ Descending complex of densities not limited above.

- Operators on $\mathcal{A}^{\bullet}\left(\mathbb{R}^{0 \mid \nu}\right)$ :

$M(\varphi): \mathcal{A}^{p}\left(\mathbb{R}^{0 \mid \nu}\right) \rightarrow \mathcal{A}^{p}\left(\mathbb{R}^{0 \mid \nu}\right)$ multiplication by a scalar function $\varphi$ $e(\varphi): \mathcal{A}^{p}\left(\mathbb{R}^{0 \mid \nu}\right) \rightarrow \mathcal{A}^{p+1}\left(\mathbb{R}^{0 \mid \nu}\right)$ by $e(\varphi)=d \varphi \wedge$

$i(\Xi): \mathcal{A}^{p}\left(\mathbb{R}^{0 \mid \nu}\right) \rightarrow \mathcal{A}^{p-1}\left(\mathbb{R}^{0 \mid \nu}\right)$ by contraction with the vectorfield $\Xi$ $\mathcal{L}_{\Xi} \equiv \mathcal{L}(\Xi):=i(\Xi) d-\operatorname{di}(\Xi)$ maps $\mathcal{A}^{p}\left(\mathbb{R}^{0 \mid \nu}\right) \rightarrow \mathcal{A}^{p}\left(\mathbb{R}^{0 \mid \nu}\right)$.

- Operators on $\mathcal{D}_{\bullet}\left(\mathbb{R}^{0 \mid \nu}\right)$ :

$M(\varphi): \mathcal{D}_{p}\left(\mathbb{R}^{0 \mid \nu}\right) \rightarrow \mathcal{D}_{p}\left(\mathbb{R}^{0 \mid \nu}\right)$, multiplication by scalar function $\varphi$ $e(\varphi): \mathcal{D}_{p}\left(\mathbb{R}^{0 \mid \nu}\right) \rightarrow \mathcal{D}_{p-1}\left(\mathbb{R}^{0 \mid \nu}\right)$ by $\mathcal{F} \mapsto d(\varphi) \cdot \mathcal{F}$

$i(\Xi): \mathcal{D}_{p}\left(\mathbb{R}^{0 \mid \nu}\right) \rightarrow \mathcal{D}_{p+1}\left(\mathbb{R}^{0 \mid \nu}\right)$ multiplication and partial symmetrization

$$
\mathcal{L}_{\Xi} \equiv \mathcal{L}(\Xi)=i(\Xi) \nabla-\nabla i(\Xi): \mathcal{D}_{p}\left(\mathbb{R}^{0 \mid \nu}\right) \rightarrow \mathcal{D}_{p}\left(\mathbb{R}^{0 \mid \nu}\right)
$$

by Lie derivative w.r.t. $\Xi$.

In section 10.2 we construct a supersymmetric Fock space. The operators $e$ and $i$ defined above can be used for representing

fermionic creation operators: $e\left(x^{m}\right)$

fermionic annihilation operators: $i\left(\partial / \partial x^{m}\right)$

bosonic creation operators: $e\left(\xi^{\mu}\right)$

bosonic annihilation operators: $i\left(\partial / \partial \xi^{\mu}\right)$.

We refer to [3] to [7] for the different definitions of graded manifolds, supermanifolds, supervarieties, superspace and sliced manifolds proposed by various people. Here we consider simply superfunctions $F$ on $\mathbb{R}^{n \mid \nu}$, that is, functions of $n$ real variables $\left\{x^{a}\right\}$ and $\nu$ Grassmann variables $\left\{\xi^{\alpha}\right\}$ :

$$
F(x, \xi)=\sum_{p=0}^{\nu} \frac{1}{p !} f_{\alpha_{1} \ldots \alpha_{p}}(x) \xi^{\alpha_{1}} \ldots \xi^{\alpha_{p}}
$$


where the functions $f_{\alpha_{1} \ldots \alpha_{p}}$ are smooth functions on $\mathbb{R}^{n}$, antisymmetric in the indices $\alpha_{1}, \ldots, \alpha_{n}$.

\subsection{Berezin integration}

A Berezin integral is a derivation

The fundamental requirement on a definite integral is expressed in terms of an integral operator $I$ and a derivative operator $D$ on a space of functions, namely

$$
D I=I D=0 .
$$

The requirement $D I=0$ for functions of real variables $f: \mathbb{R}^{D} \rightarrow \mathbb{R}$ says that the integral does not depend on the variable of integration

$$
\frac{d}{d x} \int f(x) d x=0, \quad x \in \mathbb{R} .
$$

The requirement $I D=0$ on the space of functions vanishing on their domain boundaries says

$$
\int \frac{d}{d x} f(x) d x=0
$$

This is the foundation of integration by parts

$$
0=\int d(f(x) g(x))=\int d f(x) \cdot g(x)+\int f(x) d g(x),
$$

and of the Strokes' theorem on a form $\omega$,

$$
\int_{\mathbb{M}} d \omega=\int_{\partial \mathbb{M}} \omega=0 \quad \text { since } \partial \mathbb{M} \text { is an empty set. }
$$

We shall use the requirement $I D=0$ in Section 11.1 for imposing a condition on volume elements.

We now use the fundamental requirements on Berezin integrals defined on functions $f$ of the Grassmann algebra $\Lambda_{\nu}$. The condition $D I=0$ says

$$
\frac{\partial}{\partial \xi^{i}} I(f)=0 \quad \text { for } i \in\{1, \ldots, \nu\} .
$$

Any operator on $\Lambda_{\nu}$ can be set in normal ordering $\dagger$

$$
\sum C_{K}^{J} \xi^{K} \frac{\partial}{\partial \xi^{J}}
$$

$\dagger$ This ordering is also the operator normal ordering, creation operator followed by annihilation operator, since $e\left(\xi^{\mu}\right)$ and $i\left(\partial / \partial \xi^{\mu}\right)$ can be interpreted as creation and annihilation operators (see Section 10.2). 
with $J, K$ multi-ordered indices. Therefore the condition $D I=0$ implies that $I$ is a polynomial in $\partial / \partial \xi^{i}$,

$$
I=Q\left(\frac{\partial}{\partial \xi^{1}} \cdots \frac{\partial}{\partial \xi^{\nu}}\right) .
$$

The condition $I D=0$, namely

$$
Q\left(\frac{\partial}{\partial \xi^{1}}, \ldots, \frac{\partial}{\xi^{\nu}}\right) \frac{\partial}{\partial \xi^{\mu}}=0 \text { for every } i \in\{1, \ldots, \nu\},
$$

implies

$$
I=\text { constant } \frac{\partial}{\partial \xi^{\nu}} \cdots \frac{\partial}{\partial \xi^{1}} .
$$

A Berezin integral is a derivation. The constant is a normalization constant chosen for convenience in the given context. Usual choices include $1,(2 \pi i)^{1 / 2},(2 \pi i)^{-1 / 2}$.

\section{Choosing the normalization constant}

Let $f(\xi)=\xi$, then according to (9.36)

$$
I(f)=\text { constant } \quad \text { also written } \int \xi d \xi=\int d \xi \xi=\text { constant } .
$$

The choice of constant can be dictated by the Fourier transform of the Dirac delta-function, i.e. by the Fourier transform convention (see Conventions). Here

$$
\delta(x)=\int d k \exp (2 \pi i k x)
$$

In Grassmann variables

$$
\delta(\xi)=\xi(\text { constant })^{-1} .
$$

If we write

$$
\begin{aligned}
\delta(\xi) & =\int d \kappa \exp (2 \pi i \kappa \xi)=\int d \kappa(1+2 \pi i \kappa \xi) \\
& =\operatorname{constant}(2 \pi i) \xi=\xi(\text { constant })^{-1} \xi \text { by }()
\end{aligned}
$$

then

$$
(\text { constant })^{2}=(2 \pi i)^{-1} .
$$

On the other hand, if we write

$$
\delta(\xi)=\frac{1}{2 \pi} \int d \kappa \exp (i \kappa \xi)=(2 \pi)^{-1} i \text { constant }
$$


then

$$
(\text { constant })^{2}=-2 \pi i .
$$

We could also write $\delta(\xi)=\frac{1}{2 \pi} \int \exp (i \kappa \xi) d \kappa$, then

$$
(\text { constant })^{2}=2 \pi i \text {. }
$$

Here

$$
\int d \xi \cdot \xi=(2 \pi i)^{-1 / 2}
$$

\section{Change of variable of integration}

Since integrating $f\left(\xi^{1}, \ldots, \xi^{\nu}\right)$ is taking its derivatives w.r.t. $\xi^{1}, \ldots, \xi^{\nu}$, a change of variable of integration is most easily performed on the derivatives. Given a change of coordinates $f$, we recall the induced transformations on the tangent and cotangent spaces. Let $y=f(x)$ and $\theta=f(\zeta)$;

Fig. 9.1.

$$
d y^{1} \wedge \ldots \wedge d y^{D}=d x^{1} \wedge \ldots \wedge d x^{D}\left(\operatorname{det} \frac{\partial y^{i}}{\partial x^{j}}\right)
$$

and

$$
\int d x^{1} \wedge \ldots \wedge d x^{D}(F \circ f)(x)\left(\operatorname{det} \frac{\partial f^{i}}{\partial x^{j}}\right)=\int d y^{1} \wedge \ldots \wedge d y^{D} F(y) .
$$

On the other hand, for an integral over Grassmann variables, the antisymmetry leading to a determinant is the antisymmetry of the product $\partial_{1} \ldots \partial_{D}$. And

$$
\left(\frac{\partial}{\partial \zeta^{1}} \cdots \frac{\partial}{\partial \zeta^{D}}\right)(F \circ f)(\zeta)=\left(\operatorname{det} \frac{\partial \theta^{\lambda}}{\partial \zeta^{\mu}}\right) \frac{\partial}{\partial \theta^{1}} \cdots \frac{\partial}{\partial \theta^{D}} F(\theta) .
$$


The determinant is now on the right hand side, it will become an inverse determinant when brought to the left hand side as in (9.53).

\subsection{Forms and densities}

On an ordinary manifold $\mathbb{M}^{D}$, a volume form is an exterior differential form of degree $D$; it is called a "top form" because there are no forms of degree higher than $D$ on $\mathbb{M}^{D}$ - a consequence of the antisymmetry of forms. In Grassmann calculus, forms are symmetric, there are forms of arbitrary degrees on $\mathbb{R}^{0 \mid \nu}$; therefore there are no "top forms" on $\mathbb{R}^{0 \mid \nu}$. We must look for another concept of volume element on $\mathbb{M}^{D}$ which can be generalized to $\mathbb{R}^{0 \mid \nu}$.

In the thirties [9] densities were extensively used in defining and computing integrals. Densities fell in disfavor, possibly because in contrast to forms they do not make an algebra. On the other hand, complexes (ascending and descending) can be constructed with densities as well as with forms, in ordinary and Grassmann variables.

By forms (exterior differential forms) one means totally antisymmetric covariant tensors. By densities (linear tensor densities) one means totally antisymmetric contravariant tensor-densities of weight one.

We recall properties of forms and densities on ordinary $D$-dimensional manifolds $\mathbb{M}^{D}$ which can be established in the absence of a metric tensor because they are readily useful in Grassmann calculus.

$$
\text { Ascending complex of forms on } \mathbb{M}^{D}
$$

Let $\mathcal{A}^{p}$ be the space of $p$-forms on $\mathbb{M}^{D}$ and $d$ the exterior differentiation

$$
d: \mathcal{A}^{p} \rightarrow \mathcal{A}^{p+1} .
$$

Since $d d=0$, the graded algebra $\mathcal{A}^{\bullet}$ is an ascending complex w.r.t. the operator $d$

$$
\begin{gathered}
\mathcal{A}^{0} \stackrel{d}{\longrightarrow} \mathcal{A}^{1} \stackrel{d}{\longrightarrow} \ldots \stackrel{d}{\longrightarrow} \mathcal{A}^{D} . \\
\text { Descending complex of densities on } \mathbb{M}^{D}
\end{gathered}
$$

Let $\mathcal{D}_{p}$ be the space of $p$-densities on $\mathbb{M}^{D}$ and $\nabla$ the divergence operator, also labeled $b$

$$
\nabla \cdot: \mathcal{D}_{p} \rightarrow \mathcal{D}_{p-1}, \quad \nabla \cdot \equiv b
$$

Since $b b=0, \mathcal{D}$ ( which is not a graded algebra) is a descending complex w.r.t. the divergence operator

$$
\mathcal{D}_{0} \stackrel{b}{\longleftarrow} \mathcal{D}_{1} \stackrel{b}{\longleftarrow} \ldots \stackrel{b}{\longleftarrow} \mathcal{D}_{n}
$$


Metric-dependent and dimension-dependent transformations

The metric tensor $g$ provides a correspondence $C_{g}$ between a $p$-form and a $p$-density. For instance, let $F$ by a 2 form, then

$$
\mathcal{F}^{\alpha \beta}=\sqrt{\operatorname{det} g_{\mu \nu}} F_{\gamma \delta} g^{\alpha \gamma} g^{\beta \delta}
$$

are components of a 2-density. The metric $g$ is used twice: a) raising indices, b) introducing weight 1 by multiplication with $\sqrt{\operatorname{det} g}$. This correspondence does not depend on the dimension $D$.

On an orientable manifold, the dimension $D$ can be used for transforming a $p$-density into a $(D-p)$-form. For example let $D=4$ and $p=1$

$$
t_{\alpha \beta \gamma}:=\varepsilon_{\alpha \beta \gamma \delta}^{1234} \mathcal{F}^{\delta}
$$

where the alternating symbol $\varepsilon$ defines an orientation.

The star operator (Hodge-de Rham operator, see Ref. [10], p. 295) combines the metric-dependent and the dimension-dependent transformations; it transforms a $p$-form into a $(D-p)$-form by

$$
\mathcal{T}(\omega \mid \eta)=\omega \wedge * \eta
$$

where, as usual, the scalar product of $2 p$-form $\omega$ and $\eta$ is

$$
(\omega \mid \eta)=\frac{1}{p !} \omega_{i_{1} \ldots i_{p}} \eta^{i_{1} \ldots i_{p}}
$$

and $\mathcal{T}$ is the volume element, given in example 1 below.

We shall exploit the correspondence mentioned in the first paragraph

$$
C_{g}: \mathcal{A}^{p} \rightarrow \mathcal{D}_{p}
$$

for constructing a descending complex on $\mathcal{A}^{\bullet}$ w.r.t. to the metric transpose $\delta$ of $d$ (Ref. [10], p. 296)

$$
\delta: \mathcal{A}^{p+1} \rightarrow \mathcal{A}^{p}
$$

and an ascending complex on $\mathcal{D}$.

$$
\beta: \mathcal{D}_{p} \rightarrow \mathcal{D}_{p+1}
$$

where $\beta$ is defined by the following diagram

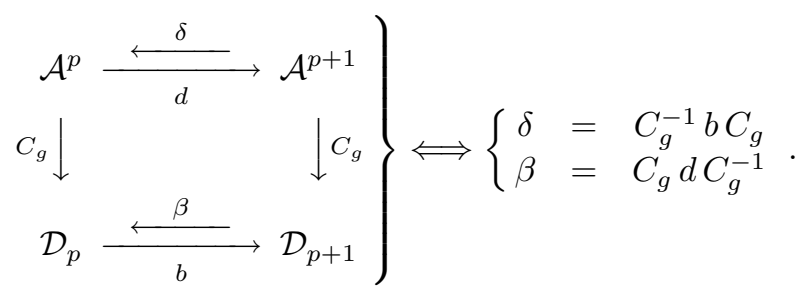


Example 1. Volume element on an oriented $D$-dimensional riemannian manifold. The volume element is

$$
\mathcal{T}:=d x^{1} \wedge \ldots \wedge d x^{D} \mathcal{I}=d x^{1} \wedge \ldots \wedge d x^{D} \sqrt{\operatorname{det} g} \text { with } \mathcal{I} \in \mathcal{D}_{0}
$$

where $\mathcal{I}$ is a scalar density corresponding to the top form

$$
d x^{1} \wedge \ldots \wedge d x^{D} \in \mathcal{A}^{D}
$$

$\mathcal{I}$ is indeed a scalar density since, under the change of coordinates $x^{\prime j}=$ $A^{j}{ }_{i} x^{i}$

$$
\mathcal{I}^{\prime}=(\operatorname{det} A) \mathcal{I}
$$

Example 2. In the thirties, the use of densities was often justified by the fact that in a number of useful examples it reduces the number of indices. For example, a vector-density in $\mathbb{M}^{4}$ can replace a 3 -form

$$
\mathcal{I}^{l}=\sqrt{\operatorname{det} g} \varepsilon_{1234}^{i j k l} t_{i j k} .
$$

An axial vector in $\mathbb{R}^{3}$ can replace a 2 -form.

\section{Grassmann forms}

Two properties of forms on real variables remain true for forms on Grassmann variables, namely

$$
\begin{gathered}
d d \omega=0 \\
d(\omega \wedge \theta)=d \omega \wedge \theta+(-1)^{\tilde{\omega} \tilde{d}} \omega \wedge d \theta
\end{gathered}
$$

where $\tilde{\omega}$ and $\tilde{d}=1$ are the parities of $\omega$ and $d$, respectively. A form on Grassmann variables is a graded totally antisymmetric covariant tensor; this means that a Grassmann $p$-form is always even.

Since a Grassmann $p$-form is symmetric the ascending complex $\mathcal{A}^{*}\left(\mathbb{R}^{0 \mid \nu}\right)$ does not terminate the $\nu$-forms.

\section{Grassmann densities}

Two properties of densities on real variables remain true for densities $\mathcal{F}$ on Grassmann variables, namely

$$
(\nabla \cdot)(\nabla \cdot) \mathcal{F}=0 .
$$

Since a density is a tensor of weight 1 , multiplication by a tensor of weight zero is the only possible product which maps a density into a density.

$$
(\nabla \cdot)(X F)=(\nabla \cdot X) \cdot F+(-1)^{\tilde{X} \tilde{\nabla}} X \nabla \cdot F, X \text { a vector field } .
$$


Since a density on Grassmann variables is a symmetric contravariant tensor; the descending complex $\mathcal{D}_{\bullet}\left(\mathbb{R}^{0 \mid \nu}\right)$ of Grassmann densities with respect to $\nabla \cdot$ does not terminate at $\nu$-densities.

\section{Volume elements}

The purpose of introducing densities was a definition of volume elements suitable both for ordinary and Grassmann variable. In example 1 ([9] p. 67) we showed how a scalar density enters a volume element on $\mathbb{M}^{D}$ and we gave the transformation ([9] p. 69) of a scalar density under a change of coordinates in $\mathbb{M}^{D}$. But for generalizing scalar densities to Grassmann volume element, we start from Pauli's definition ([11] p. 32) which follows Weyl's terminology ([12] p. 109). "If $\int \mathcal{F} d x$ is an invariant [under a change of coordinate system] then $\mathcal{F}$ is called a scalar density".

Under the change of variable $y(x)$

$$
\mathcal{F}^{\prime}=\operatorname{det}\left(\partial y^{j} / \partial x^{i}\right) \mathcal{F} .
$$

If the Berezin integral

$$
\int d \xi^{\nu} \ldots d \xi^{1} f\left(\xi^{1}, \ldots, \xi^{\nu}\right)=\frac{\partial}{\partial \xi^{\nu}} \ldots \frac{\partial}{\partial \xi^{1}} f\left(\xi^{1}, \ldots, \xi^{\nu}\right)
$$

is invariant under the change of coordinates $\theta(\xi)$ then $f$ is a Grassmann scalar density. It follows from the formula for change of variable of integration (9.38) that a Grassmann scalar density is a scalar divided by $\operatorname{det}\left(\partial \theta^{\lambda} / \partial \xi^{\mu}\right)$

$$
f^{\prime}=\left(\operatorname{det}\left(\partial \theta^{\lambda} / \partial \xi^{\mu}\right)\right)^{-1} f
$$




\section{Bibliography}

[1] F.A. Berezin, The method of Second Quantization (in Russian Nauka, Moscow, 1965; English translation Academic Press, New York 1966).

[2] F.A. Berezin and M.S. Marinov, Classical spin and Grassmann algebra, JETP Lett. 21 (1975), 320-321.

F.A. Berezin and M.S. Marinov, Particle spin dynamics as the Grassmann variant of classical mechanics, Annals of Physics 104 (1977), 336-362.

M.S. Marinov, Path integrals in quantum theory, Physics Reports 60 (1980), 1-57.

[3] P. Cartier, C. DeWitt-Morette, M. Ihl, and Ch. Sämann, Appendix by M.E. Bell, Supermanifolds - Applications to supersymmetry (arXiv:math-ph/0202026 v1 19 Feb 2002).

Marinov Memorial Volume Multiple Facets of Quantization and Supersymmetry, Eds M. Olshanetsky and A. Vainshtein, 2002, pp. 412-457.

[4] B. DeWitt, Supermanifolds (Cambridge University Press, Second Edition 1992). In this book complex conjugation is defined $\left(z z^{\prime}\right)^{*}=z^{\prime *} z^{*}$.

[5] Seminar on Supermanifolds, known as sos, over 2000 pages written by D. Leites, colleagues, students and collaborators from 1977 to 2000.

[6] Yvonne Choquet-Bruhat, Graded Bundles and Supermanifolds (Bibliopolis, Naples 1989).

[7] T. Voronov, Geometric Integration Theory on Supermanifolds, Sov. Sci. Rev. C. Math. Phys. 9 (1992), 1-138.

[8] M.E. Bell, Introduction to Supersymmetry (Master Thesis, University of Texas 2002).

[9] L. Brillouin, Les tenseurs en mécanique et en élasticité (Masson, Paris 1938).

[10] Yvonne Choquet-Bruhat, C. DeWitt-Morette, Analysis, Manifolds, and Physics, Part I: Basics (with M. Dillard-Bleick), Rev. Ed. (North Holland, Amsterdam, 1996); Part II, Revised and Enlarged Ed. (North Holland, Amsterdam, 2000).

[11] W. Pauli, Theory of Relativity (Pergamon Press New York 1958); translated with supplementary notes by the author from "Relativitätstheorie" in Encyclopädie der matematischen Wissenschaften, Vol. 5, Part 2, pp. 539-775 (B.G. Teubner, Leipzig, 1921). 
[12] H. Weyl, Raum-Zeit-Materie (Julius Springer, Berlin, 1921) [English translation: Space-Time-Matter (Dover, New York, 1950)]. The word "skew" is missing in the English translation of schiefsymmetrischen.

[13] G. Roepstorff, Path Integral Approach to Quantum Physics, An Introduction. Springer Verlag, 1994. 